Revista de Psicología de la Salud, 1989, Volumen 1, N. 1, Pág. 127 a 145

\title{
EL SINNDROME DE INMUNODEFICIENCIA ADQUIRIDA (SIDA) COMO ESTRESOR EN MEDICOS Y ENFERMERAS DE HOSPITAL
}

\section{Abilio Reig y Agustín Caruana}

\author{
Departamento de Psicología de la Salud. \\ Facultad de Medicina. Universidad de Alicante
}

\section{RESUMEN}

En este capítulo hemos analizado el grado de estrés percibido asociado a la posibilidad de tener pacientes con SIDA en 93 profesionales de la salud que trabajan en hospitales. El $32 \%$ de la muestra asocia unos niveles altos de tensión a esa posibilidad. No existe una diferencia significativa entre médicos y enfermeras en cuanto a estrés percibido asociado al SIDA, mostrándose como poco relevantes las variables sociodemográficas en la determinación del nivel de estrés. Los estresores asociados a niveles altos de tensión en relación al SIDA tienen que ver con aspectos como la probabilidad de contagio nosocomial casual, no poder resolver con satisfacción los problemas que se plantean, tener que informar, ver de cerca la muerte, mantener la confianza en uno mismo e inspirar confianza en los pacientes, y los derivados de las complicaciones en el tratamiento. Ambos grupos presentan, además, estresores especificos diferentes. En los médicos, aquellos como presentar casos a sesiones clínicas, decidir qué aspectos son prioritarios, el trabajo agotador, hablar con los pacientes, etc.; mientras que en enfermería se circunscriben más a nivel asistencial (atender en situaciones de urgencia, o a pacientes moribundos/terminales, o graves). Con todo, mediante análisis de regresión múltiple, comprobamos que es en el grupo de médicos donde la posibilidad de contagio nosocomial constituye la principal causa de estrés asociado a tener pacientes con SIDA (coef. regresión estándar: 0.45), mientras que el peso es menor en enfermería: posibilidad de contagio $(0,35)$, tener que informar $(0.33)$, asistencia a casos urgentes $(0.31)$, que son coef, de regresión estandarizados con niveles de significación bajos.

\section{ABSTRACT}

Acquired immune-deficiency syndrome (AIDS) appears to elicit fearful and prejudicial attitudes in some health professionals. In the study reported here, 93 health professionals, 42 doctors and 51 nurses, working in hospitals, were asked about the level of pressure they associate to the possibility of having to treat AIDS patients. Thirty two percent of the subjects associated high levels of pressure to that possibility. No statistical differen- 
ce was found between physicians and nurses, and demographic variables were not related to stress level. High levels of pressure in treating AIDS patients were associated to stressors such as dealing with patients with an infectious disease, not being able to solve the problems they are faced with, having to inform patients, exposure to patients's de-ath, maintaining self-confidence, having to inspire trust and confidence in patients, and those which arise from complications in treatment. Furthermore, doctors and nurses show different specific stressors. Doctors attain higher scores in pressure on stressors such as keeping up with the scientific literature, deciding priorities, finding their job physically exhausting, etc. Nurses show higher scores in pressure on stressors such as assisting an urgent case, or looking after severe ill patients. Multiple regression analysis was used to analyze the relationship between the dependent variable "Treating AIDS patients", and the set of independent variables (demographic, stressors). The most powerful predictor in the level of pressure associated to treating AIDS patients was the risk of being infected.

\section{INTRODUCCION}

El síndrome de inmunodeficiencia adquirida (SIDA), tal como ha sido definido por los Centros de Control de la Enfermedad (CDC) de EEUU, consiste en la aparición de una enfermedad que permite sospechar, al menos moderadamente, la existencia de un defecto de la inmunidad celular y que se manifiesta en una persona, sin que medie causa conocida que justifique esa disminución de la resistencia ante las enfermedades (Goedert \& Blattier, 1986). Esta alteración de los mecanismos de defensa del huésped predispone a las infecciones provocada por gérmenes oportunistas (Pneumocystis carinil, micobacterias atípicas, o cryptococcus neoformans), así como a la aparición de sarcoma de Kaposi, o a linfomas no Hodgkin. Este defecto de la inmunidad celular se caracteriza por anergia cutánea, disminución del número de células $\mathrm{T}$, y descenso de la relación de las subpoblaciones de OKT4 (helper-inductors) con respecto a los de OKT8 (supresores-citotóxicos). Se suele denominar CRS (complejo relacionado con el SIDA) al síndrome que puede preceder al SIDA y que se caracteriza por fatiga, fiebre, sudoración nocturna, diarrea, candidiasis oral, pérdida de peso y linfadenopatías. Hoy sabemos con seguridad que el agente causante del SIDA es el virus linfotrópico T-III humano (HTLV-III), también denominado virus asociado a linfadenopatía (LAV). Al objeto de superar estas diferencias de denominación (HTLV-III/LAV) actualmente se emplea el término más correcto de virus de la inmunodeficiencia humana-1 $(\mathrm{VIH}-1)$. La deficiencia inmunológica es sólo uno de los efectos del HIV-1, ya que también desencadena graves problemas de salud relacionados con el sistema nervioso central (Wolcott, 1986; Detmer \& Lu,1986-87; Burton, 1987). 
Hasta el 25 de mayo de 1987 se han comunicado a la OMS 60.405 casos de SIDA distribuidos en 121 paises, aunque se cree que pueden ser más de 100.000 (Delgado, 1988; Mann, 1987). En todo caso, Mann considera que pueden existir en todo el mundo unos 10 millones de personas infectadas por $\mathrm{VIH}-1$ que aún no han presentado la enfermedad. En Europa, hasta marzo de 1987, se han registrado 5687 casos de SIDA (Duvic, Rios, \& Brewston, 1987), siendo el número de casos en España, según el Ministerio de Sanidad y Consumo, de 508 con fecha 26 de junio de 1987.

Desde el año 1981, en que por primera vez se diagnosticó, el SIDA ha presentado, fundamentalmente, un fuerte impacto médico, psicológico, económico y social (Bennett, 1987; Velimirovic, 1987). En tan pocos años ha sido sorprendente el progreso biomédico realizado: identificación del virus, ensayos para detectar el virus, vías de transmisión, avances terapéuticos moderadamente prometedores, y el comienzo del desarrollo de una vacuna ( Tor, 1987; Gallo, 1987a, 1987b; Selwyn,1987). Han aparecido diversos trabajos sobre el impacto psicológico del SIDA, o su diagnóstico de seropositividad, en los pacientes (Christ \& Wiener, 1986; Gold, Seymour \& Sahl, 1986), las estrategias de afrontamiento utilizadas (Martin \& Vance, 1984; Coates, Temoshok \& Mandel, 1984; Ferrara, 1984; Morin, Charles \& Malyon, 1984 ), la aparición de una nueva variedad de fobia, la sidafobia (Fonseca, 1988), las medidas preventivas a adoptar (OMS, 1987), y se ha cuantificado el tremendo gasto económico que está suponiendo (Hardy, Ranch, Echenberg, Morgan \& Curran, 1986). Concretamente en Europa se ha calculado el coste medio de un paciente con SIDA en 112.500 ecus, unos 16.000 .000 de pesetas. En un artículo de hace algunos años, Batchelor aseguraba que el SIDA se había convertido en una emergencia psicológica y de salud pública (Batchelor, 1984), ya que sin un tratamiento o cura eficaz la única esperanza de parar la epidemia era a través de la prevención, vertiente esta en la que las ciencias sociales y del comportamiento ofrecen la mejor perspectiva para propiciar un cambio de comportamientos individuales y grupales. De hecho, debido a la ausencia de un tratamiento eficaz para el SIDA (pese a los prometedores resultados actuales con la zidovudina), se han intensificado los esfuerzos en el campo preventivo (Bayés, 1986). Así, las medidas técnicas de detección de los hemoderivados, y el tratamiento mediante calor de los concentrados de factores séricos, esta evitando que se transmita el virus a pacientes hemofilicos (CDC, 1987). Se ha avanzado menos en la realización de campañas educacionales sobre actividades específicas de riesgo, que sean eficaces y accesibles, tanto para "grupos" de riesgo como para la población en general. 
También se esta prestando poca atención a la repercusión psicosocial del SIDA en el personal sanitario que tiene que atender a dichos pacientes. Diversos trabajos han investigado las manifestaciones de estrés en aquellos profesionales de la salud que tienen que trabajar diariamente con estos enfermos (O'Donnell, O'Donnell, Pleck, Snarey \& Rose, 1987). Entre estos profesionales se han señalado como situaciones frecuentes la desmoralización (Gascón, 1987), la sobrecarga psicológica y física, el estrés, la ansiedad, la depresión (Watcher,1986; Horstman \& McKusick,1986), los miedos al contagio, y temores en la dispensa de cuidados, (Blumenfield, Smith, Milazzo, Seropian, \& Wormser,1987; Wachter,1987), aunque también otros autores han señalado un incremento en algunos aspectos positivos, como una mayor estimulación intelectual y una mayor satisfacción con la carrera (profesión) (Horstman \& McKusick,1986).

Diversas circunstancias hacen que trabajar con pacientes, o potenciales pacientes con SIDA, sea especialmente estresante para los profesionales de la salud. De una parte encontramos situaciones tales como la falta de una inmunoterapia vírica específica, o la naturaleza contagiosa devastadora, desordenada y con múltiples complicaciones de este problema de salud, que requieren, generalmente, un tipo de asistencia profesional caracterizado por un trabajo intensivo y de gran dedicación, con sobrecarga emocional y física. A menudo, como ha señalado Selwyn (1987), no existe otra opción para los clínicos que observar cómo sufren y fallecen estos jóvenes a pesar de sus esfuerzos para salvarlos, y tras el fracaso de todos sus intentos terapéuticos. De otra parte, aparecería el miedo al contagio nosocomial por un contacto casual, el tener que afrontar situaciones nuevas, informar a pacientes, familiares, o autoridades; estar preparado para controlar las situaciones de estrés derivadas de la notificación del resultado positivo en las pruebas, o las que vayan surgiendo a lo largo de la enfermedad, así como los problemas diarios con el personal no sanitario para que mantengan la limpieza, $u$ otros servicios, en las habitaciones de los pacientes. Además, en muchos casos, obliga a los profesionales de la salud a explorar su grado de aversión a los toxicómanos, o su grado de homofobia. Así, en estudiantes de medicina se ha encontrado actitudes negativas, de miedo y prejuiciosas hacia pacientes con SIDA u homosexuales (Kelly, Lawrence, Smith, Hood \& Cook, 1987), corroborándose estas mismas actitudes en médicos (Kelly, Lawrence, Smith, Hood \& Cook, 1987; Mathews, Booth, Turner \& Kessler, 1987) y en personal de hospital (O'Donell, O'Donell, Pleck, Snarey \& Rose, 1987; Douglas, Kalman \& Kalman, 1985). Todo ello sin olvidar, además, la posibilidad real de que el propio profesional de la salud sea seropositivo al HIV (Vaqué \& Arcalis, 1987). 
El estudio del impacto que los pacientes con SIDA tienen sobre el personal sanitario es entre otras cosas, a que los profesionales de la salud tienen en sus manos la posibilidad de contribuir e influir en la salud física y mental de estos pacientes. Su apoyo es crítico para ayudar a las familias de los pacientes a superar los desafíos de la enfermedad, y deben estar capacitados para la difusión de la información acerca de la enfermedad a la población. El estudio de sus comportamientos y actitudes ante el SIDA, sin duda facilitará el desarrollo de programas de entrenamiento, que hagan posible la mejora cuantitativa y cualitativa del servicio asistencial a los pacientes con SIDA, así como la reducción del posible estrés laboral asociado.

En el trabajo que presentamos a continuación hemos intentado analizar la circunstancia, potencial o real, de tener en el hospital pacientes con el síndrome de inmunodeficiencia adquirida, como posible estresor laboral en profesionales de la salud que trabajan en hospitales generales. Asimismo analizamos qué variables pueden ser responsables del grado de tensión asociado a este problema de salud.

\section{METODO}

Esta investigación se enmarca dentro de un proyecto más amplio de estrés laboral en profesionales de la salud. El instrumento que hemos utilizado consiste en un cuestionario de autocalificación de 157 situaciones potencialmente estresantes diseñado al efecto (Reig y Caruana, 1987). Una de estas situaciones corresponde a tener pacientes con SIDA, y se debla contestar eligiendo una de las 5 alternativas de respuesta en base al grado de tensión asociado al item: nada (1), leve (2), moderada (3), bastante (4), mucha (5).

Con la finalidad de realizar un estudio piloto acerca de las posibles situaciones estresantes repartimos un total de 300 cuestionarios, con hoja de instrucciones y datos sociodemográficos, para su cumplimentación voluntaria y anónima en los hospitales del Insalud de Alicante y Elche. La tasa de respuesta ha sido de 93 cuestionarios correctamente cumplimentados, lo que supone el $31 \%$. El hecho de coincidir el inicio de la investigación con una época de huelgas en el sector -primavera del 87- puede haber tenido algún tipo de repercusión en el porcentaje precedente.

Los datos han sido procesados en el Centro de Cálculo de la Universidad de Alicante.

\section{RESULTADOS}

Han participado en este trabajo un total de 93 profesionales de la 
Tabla número 1: DESCRIPCION DE LA MUESTRA.

\begin{tabular}{|c|c|c|c|c|}
\hline \multirow[b]{2}{*}{ EDAD } & \multicolumn{2}{|c|}{$\begin{array}{l}\text { ENFERMERAS } \\
\quad(n=51)\end{array}$} & \multicolumn{2}{|l|}{$\begin{array}{l}\text { MEDICOS } \\
(n=42)\end{array}$} \\
\hline & $\begin{array}{c}x \\
35.87\end{array}$ & $\begin{array}{l}\text { d.t. } \\
8.39\end{array}$ & $\begin{array}{c}x \\
33.53\end{array}$ & $\begin{array}{l}\text { d.t. } \\
6.61\end{array}$ \\
\hline SEXO & Varones & Mujeres & Varones & Mujeres \\
\hline$(\%)$ & 32 & 68 & 90.5 & 9.5 \\
\hline $\begin{array}{l}\text { ESTADO } \\
\text { CIVIL } \\
(\%)\end{array}$ & $\begin{array}{l}\text { Cas. Sol. } \\
62.7 \quad 29.4\end{array}$ & Div. & $\begin{array}{l}\text { Cas. } \\
73\end{array}$ & Div. \\
\hline $\begin{array}{l}\text { ESPECIA- } \\
\text { LIDAD } \\
(\%)\end{array}$ & SI & NO & SI & NO \\
\hline $\begin{array}{l}\text { EXPE- } \\
\text { RIENCIA } \\
\text { PROFESIONAL }\end{array}$ & \multicolumn{2}{|c|}{$\begin{array}{l}\text { Rango Medio } \\
\text { Entre } 8 \text { y } 11 \text { años }\end{array}$} & \multicolumn{2}{|c|}{$\begin{array}{l}\text { Rango Medio } \\
\text { Entre } 8 \text { y } 11 \text { años }\end{array}$} \\
\hline $\begin{array}{l}\text { TURNOS } \\
(\%)\end{array}$ & Mañ. Tar. & Noch. Rot. & $\begin{array}{l}\text { Mañ. Tar. } \\
90.5 \quad 2.4 \\
\end{array}$ & Noch. Rot. \\
\hline $\begin{array}{l}\text { HORAS DE } \\
\text { TRABAJO POR } \\
\text { SEMANA }\end{array}$ & \multicolumn{2}{|c|}{ Rango Medio } & \multicolumn{2}{|c|}{ Rango Medio } \\
\hline $\begin{array}{l}\text { PLAZA } \\
(\%)\end{array}$ & $\begin{array}{l}\text { Fija } \\
82.4\end{array}$ & 17.6 & Fija & 43 \\
\hline $\begin{array}{l}\text { PACIENTES } \\
\text { QUE VEN } \\
\text { HABITUAL- } \\
\text { MENTE }\end{array}$ & 30.35 & 7.57 & 18.72 & 12.84 \\
\hline CATEGORIA & \multicolumn{2}{|c|}{$\begin{array}{l}44 \text { ENF. planta } \\
7 \text { Supervisoras/es }\end{array}$} & \multicolumn{2}{|c|}{$\begin{array}{l}8 \text { residentes } \\
25 \text { adjuntos } \\
5 \text { Jefes de Sección } \\
3 \text { Jefes de Servicio }\end{array}$} \\
\hline
\end{tabular}


Figura 1. Grado de tensión (\%) asociado a tener pacientes con SIDA

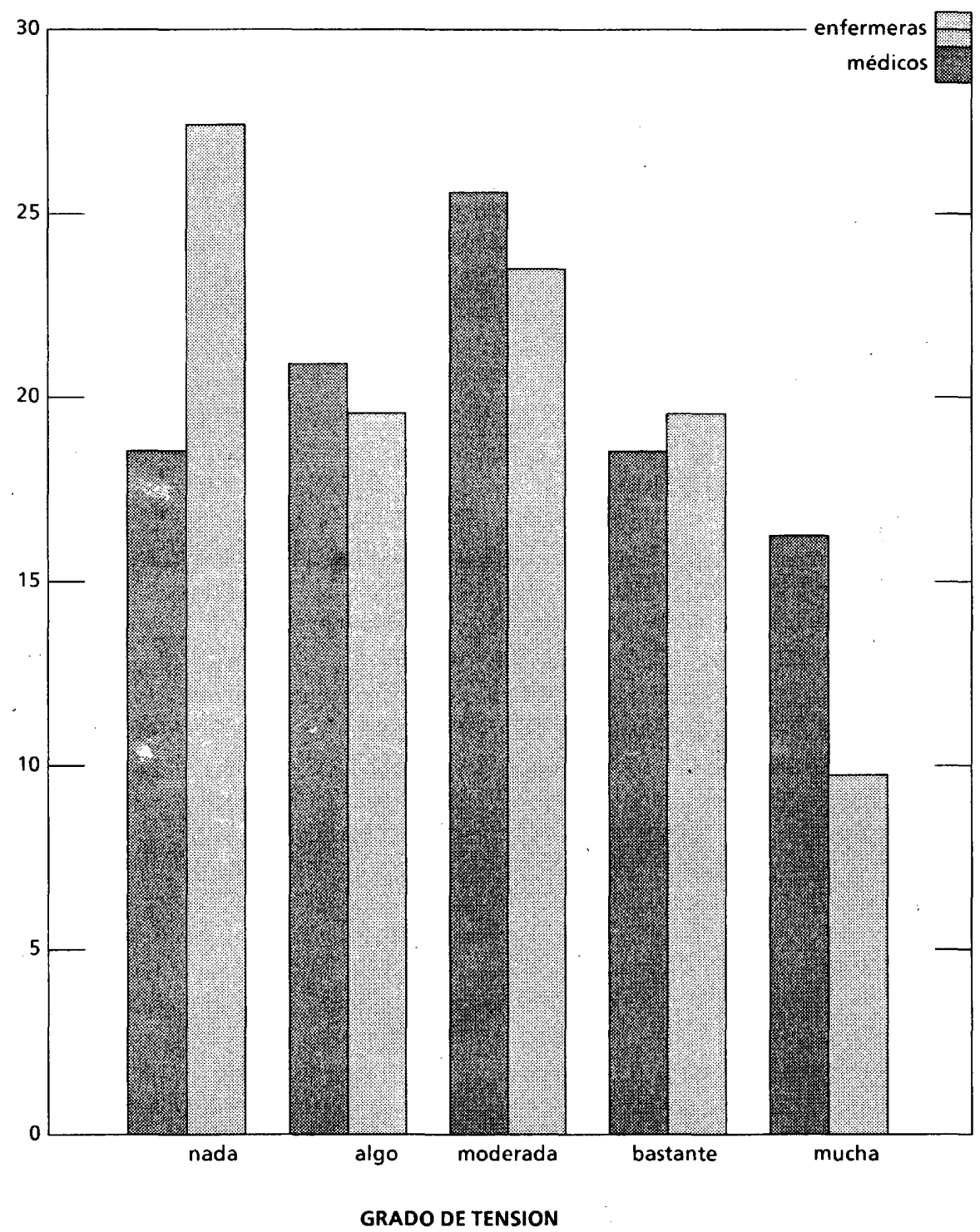


salud (51 D.U.Es y 42 médicos) que trabajan en hospitales del Insalud de la provincia de Alicante. El grupo de médicos está integrado por 38 varones (el $90.5 \%$ ) y $4(9.5 \%)$ mujeres, mientras que el de enfermería lo forman 35 mujeres (68\%) y 16 varones (32\%). La edad media de los DUEs es de 35.87 años (d.t. $=8.39$ ), y la de los médicos de 33.53 (d.t. $=6.61$ ). Otros datos sociodemográficos de interés los presentamos en la tabla número 1.

El tener pacientes con SIDA produce a los médicos un grado de tensión moderado $(\bar{X}=2.95$; d.t. $=1.36)$, mientras que en el grupo de enfermería el grado de tensión es algo menor $(\bar{X}=2.64$; d.t. = 1.33). En la figura 1 mostramos la distribución de los porcentajes, tanto para médicos como para enfermeras, en relación al grado estimado de tensión. En conjunto, el $\mathbf{2 3 . 4 0 \%}$ de la muestra señala no experimentar nada de tensión asociado a tener pacientes con SIDA, el $20.21 \%$ manifiesta tener un grado leve de tensión, el $24.46 \%$ un grado moderado, el $19.14 \%$ bastante tensión, y el $12.76 \%$ indica mucha tensión.

El valor $\mathrm{Chi}^{2}$ de Pearson que aparece es de 1.61, lo que con 4 grados de libertad se asocia a una probabilidad de 0.80 , indicativo de una distribución de porcentajes uniforme, sin diferencias estadísticamente significativas entre enfermeras y médicos. La prueba de diferencias entre medias $t$ de Student arroja un valor de 1.15, indicativo, asimismo, de la ausencia de una diferencia significativa entre el grado de tensión asociado al SIDA entre médicos y enfermeras.

En el grupo de médicos no aparece ninguna relación significativa entre el grado de tensión asociado a tener pacientes con SIDA y las variables sociodemográficas estudiadas (ver tabla número 2). No obstante, en el grupo de enfermeras aquellas que tienen más años de experiencia profesional señalan un nivel de tensión algo mayor $(r=0.32)$, así como aquellas que realizan menos horas de trabajo semanal $(r=0.31)$. Ambas asociaciones, sin embargo, sólo son significativas al $5 \%$.

Tabla número 2.: Análisis relacional ( $r$ de Pearson) entre el grado de tensión asociado a tener pacientes con SIDA y variables sociodemográficas.

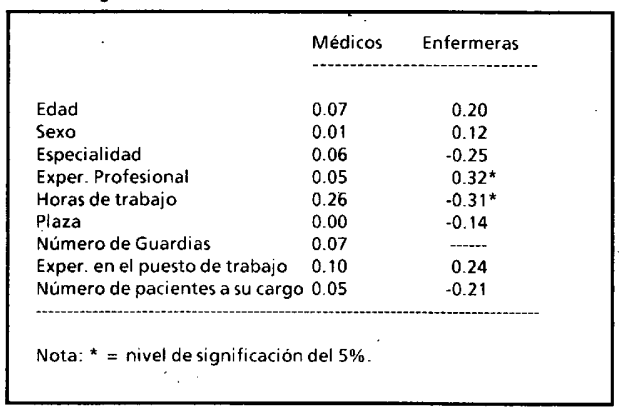


Al objeto de averiguar las posibles implicaciones con otros tipos de estresores presentamos las correlaciones en la tabla 3. Como podemos apreciar en esta tabla, los médicos ,frente a las enfermeras, presentan una mayor problemática tensional asociada a tener pacientes con SIDA. Fundamentalmente, tanto en médicos como en enfermeras, los estresores asociados al SIDA tienen que ver con la posible situación de contagio, con el hecho de tener que informar a los pacientes, o tener que informar de noticias negativas, ver de cerca la muerte, no poder resolver con satisfacción los problemas que se plantean, mantener la confianza, tanto con uno mismo como en relación a los pacientes, y el estrés derivado de las complicaciones en el tratamiento.

Además de estas importantes fuentes de estrés, unidas a la posible amenaza de posibles denuncias, los médicos presentan niveles de tensión asociados al SIDA en relación a no ver los frutos de su esfuerzo, decidir qué aspectos son prioritarios, afrontar situaciones nuevas, no ver mejoría en los pacientes, tratar con gente agresiva, o tener que presentar casos a sesiones clínicas.

Sin embargo, con la finalidad de explorar con mayor precisión las contribuciones únicas de estos estresores laborales, hemos sometido a análisis de regresión múltiple aquellos items significativamente más implicados en la situación de tensión por tener pacientes con SIDA (tablas números 4 y 5 ). En la muestra de médicos, la aportación de los 15 estresores estudiados arroja una correlación múltiple de 0.86 (con un error estandar de 0.89 ), y una correlación múltiple al cuadrado de 0.73 . El análisis de varianza presenta una razón $F$ de 4.43 , significativa al 0.0006 , lo que indica que el coeficiente de correlación múltiple al cuadrado es significativo.

El único estresor, como podemos ver en la tabla, que aparece con un coeficiente de regresión estandarizado significativo es "tener pacientes que me puedan contagiar una enfermedad" $(t=2.68, p=$ 0.0129 ). En otras palabras, de las fuentes de tensión implicadas en los casos de SIDA, parece ser que, en los médicos, el miedo al contagio es la única que explica la mayor parte de la varianza del posible estrés derivado de tener pacientes con SIDA.

En el caso del personal de enfermería, con una probabildad menor del $10 \%$ (dos colas), aparecen tres estresores asociados: la atención en situaciones de urgencia $(0.31 ; t=1.98, p=0.0548)$, el posible contagio $(0.34 ; t=1.89, p=0.0654)$, y la información al paciente $(0.33$; $t=1.70, p=0.09$ )

En conjunto, los 10 estresores analizados en el personal de enfermería presentan una $r$ múltiple de 0.66 (error de estimación estandar de 1.10), y una $r^{2}$ múltiple de 0.44. En el análisis de varianza 
Tabla número 3: Análisis relacional entre el grado de tensión asociado a tener pacientes con SIDA y la tensión asociada a los items que a continuación se especifican.

\section{Médicos Enfermeras}

Tener pacientes que me puedan con-

tagiar una enfermedad

$\begin{array}{ll}0.72^{\star \star \star} & 0.55^{\star \star} \\ 0.56^{\star \star \star} & - \\ 0.54^{\star \star \star} & 0.29^{\star} \\ 0.54^{\star \star \star} & -\cdots \\ 0.54^{\star \star \star} & --- \\ 0.53^{\star \star \star} & 0.30^{\star} \\ 0.52^{\star \star \star} & 0.50^{\star \star \star} \\ 0.52^{\star} & -\cdots \\ & \\ 0.52^{\star \star \star} & -\cdots\end{array}$

No ver los frutos de mi esfuerzo $\ldots . . .0 .56^{\star \star \star}$

Ver de cerca la muerte $\ldots \ldots \ldots \ldots .0 .54^{\star \star \star}$

Tratar con gente agresiva $\ldots \ldots \ldots .0 .54^{\star \star \star}$

Presentar casos en sesiones clínicas .. $0.54^{\star \star \star}$

Tener que dar una mala noticia $\ldots . . .0 .53^{\star \star *}$

Tener que informar a los pacientes.

Decidir que aspectos son prioritarios

El trabajo que realizo es físicamente agotador

$0.52^{\star \star \star}$

No poder resolver los problemas que se me plantean

$0.51^{\star \star *}$

Tener que recurrir a otros servicios o

especialistas para poder realizar mi

$\begin{array}{ccc}\text { trabajo } \ldots \ldots \ldots \ldots \ldots \ldots \ldots \ldots & 0.47^{\star \star} \\ \text { Pacientes que no cooperan } \ldots \ldots \ldots & 0.46^{\star \star}\end{array}$

Tener que tomar una decisión en

casos delicados

$0.44^{\star \star}$

Que me amenacen con denunciarme

$0.44^{\star \star}$

0.37 *

Hablar con los pacientes

$0.43^{* *}$

Afrontar situaciónes nuevas

$0.42^{\star \star}$

Mantener la confianza en mí mismo

$0.27^{\star}$

$0.42^{\star \star}$

Pacientes que no mejoran con el tra-

tamiento

$0.41 * \star$

Tener que inspirar seguridad y con-

fianza a los pacientes

$0.32^{\star}$

$0.29^{\star}$

Que surgan complicaciones en el tra-

tamiento

$0.31^{\star}$

$0.29^{*}$

Asistir a un caso urgente

$0.29 *$

Cuidar a pacientes moribundos/termi-

nales

$0.29 *$

Pacientes graves

$0.27^{\star}$

Nota. niveles de significación: ${ }^{\star}=5 \% ;{ }^{\star \star}=1 \% ;{ }^{\star \star}{ }^{\star}=0.1 \%$. 
Tabla número 4: Análisis de regresión múltiple con el personal médico utilizando como variable dependiente la situación de tener pacientes con SIDA.

\begin{tabular}{|c|c|c|c|}
\hline Variables independientes & $\begin{array}{l}\text { Coeficiente } \\
\text { de regresión } \\
\text { estandar }\end{array}$ & $\mathrm{t}$ & prob \\
\hline $\begin{array}{l}\text { Pacientes que me puedan con- } \\
\text { tagiar una enfermedad ............ }\end{array}$ & 0.49 & 2.68 & 0.01 \\
\hline Ver de cerca la muerte ............... & 0.20 & 1.26 & 0.22 \\
\hline Tratar con gente agresiva .......... & 0.10 & 0.58 & 0.56 \\
\hline $\begin{array}{l}\text { Tener que dar una mala noti- } \\
\text { cia }\end{array}$ & 0.21 & 1.00 & 0.33 \\
\hline $\begin{array}{l}\text { Tener que informar a los pa- } \\
\text { cientes }\end{array}$ & 0.10 & 0.56 & 0.58 \\
\hline $\begin{array}{l}\text { No poder resolver los proble- } \\
\text { mas que se me plantean.......... }\end{array}$ & 0.20 & 1.01 & 0.32 \\
\hline $\begin{array}{l}\text { Tener que tomar una decisión } \\
\text { en casos delicados }\end{array}$ & -0.23 & -0.81 & 0.42 \\
\hline Hablar con los pacientes............. & 0.05 & 0.28 & 0.78 \\
\hline Afrontar situaciónes nuevas........ & 0.02 & 0.13 & 0.89 \\
\hline 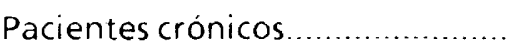 & -0.39 & -0.22 & 0.83 \\
\hline $\begin{array}{l}\text { Pacientes que no mejoran con } \\
\text { el tratamiento }\end{array}$ & 0.05 & 0.31 & 0.76 \\
\hline Que los pacientes me cuenten & & & \\
\hline $\begin{array}{l}\text { sus problemas............................. } \\
\text { Tener que tomar decisiones }\end{array}$ & -0.06 & -0.36 & 0.72 \\
\hline $\begin{array}{l}\text { drásticas en casos urgentes........ } \\
\text { No ver los frutos de mi esfuer- }\end{array}$ & -0.11 & -0.52 & 0.61 \\
\hline 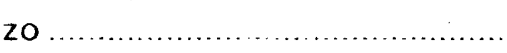 & -0.07 & -0.37 & 0.72 \\
\hline $\begin{array}{l}\text { Decidir que aspectos son prio- } \\
\text { ritarios }\end{array}$ & 0.23 & 1.34 & 0.19 \\
\hline
\end{tabular}

aparece una razón $\mathrm{F}$ de 2.96 significativa al 0.0076 .

\section{DISCUSION}

Hemos comprobado, a la luz de los resultados obtenidos, que el hecho o la posibilidad de tener pacientes con SIDA se asocia a un grado moderado de tensión en los profesionales de la salud estudiados. Con todo, hay que resaltar que un tercio del total de profesionales de la muestra (el $31.9 \%$ ) señala un estrés alto (categorías bastante y mucha). El dato de que los grados de tensión no difieran significati- 
vamente entre médicos y enfermeras, y que, básicamente, no se asocien a variables sociodemográficas, sugiere la posibilidad de llevar a cabo, en los casos que así se requiera, programas con paquetes educativos uniformes.

Los datos suministrados por los análisis de regresión muestran que la posibilidad de contagio del virus es la fuente más importante de estrés que suscita el SIDA en el profesional de la salud de un hospital general. No obstante, el relativo escaso número de profesionales que han participado en este estudio piloto, y el hecho de que en estos hospitales no se estaba tratando - en el momento de hacer el estudio- un numeroso grupo de pacientes con SIDA (1), nos obliga, en todo caso, a realizar conclusiones cautelosas. Pensamos, no obstante, que es necesario seguir realizando este tipo de investigaciones al objeto de explorar y detectar las posibles causas de resistencia, que puedan impedir a estos profesionales ofrecer una óptima prestación y calidad asistencial.

Tabla número 5: Análisis de regresión múltiple con el grupo de personal de enfermería utilizando como variable dependiente la situación de tener pacientes con SIDA.

\begin{tabular}{|c|c|c|c|}
\hline Variables independientes & $\begin{array}{l}\text { Coeficiente } \\
\text { de regresión } \\
\text { estandar }\end{array}$ & $\mathrm{t}$ & prob. \\
\hline $\begin{array}{l}\text { Pacientes que me puedan con- } \\
\text { tagiar una enfermedad.......... }\end{array}$ & 0.35 & 1.90 & 0.06 \\
\hline $\begin{array}{l}\text { Ver de cerca la muerte .......... } \\
\text { Tener que dar una mala noti- }\end{array}$ & 0.11 & 0.59 & 0.56 \\
\hline cia .................................... & -0.06 & -0.32 & .0 .75 \\
\hline $\begin{array}{l}\text { cientes .............................. } \\
\text { No poder resolver los proble- }\end{array}$ & 0.33 & 1.70 & 0.09 \\
\hline mas que se me plantean ........ & 0.15 & 0.85 & 0.40 \\
\hline Quejas de los pacientes ........ & -0.13 & -0.91 & 0.37 \\
\hline $\begin{array}{l}\text { Pacientes graves ................. } \\
\text { Tener què consolar a los fami- }\end{array}$ & -0.18 & -1.00 & 0.32 \\
\hline liares de los pacientes .......... & 0.12 & 0.75 & 0.45 \\
\hline dos/terminales ...................... & -0.26 & -1.39 & 0.17 \\
\hline
\end{tabular}


O'Donnell et al. (1987) han podido comprobar, con una muestra de 237 trabajadores de un hospital que habla admitido 60 casos de SIDA, que a mayor contacto con pacientes de SIDA, los profesionales de la salud presentaban niveles de estrés percibido más bajo $(r=0.25$, prob. $=0.01)$, no dándose esta tendencia en aquellos trabajadores con contactos frecuentes pero impersonales (O'Donnell et al. 1987). Estos autores señalan, además, que los trabajadores del hospital solían exagerar la cantidad de tiempo que dedicaban al cuidado de estos pacientes, y que consideraban este tiempo de cuidado como uno de los aspectos más estresantes de su trabajo. Un $24 \%$ de estos trabajadores afirmaba encontrarse en un riesgo elevado de infectarse a causa de su trabajo, y el $35.5 \%$ señalaba encontrarse incómodo con estos pacientes. Además, el $58 \%$ señalaba no tener el conocimiento suficiente para hacer frente a los problemas emocionales que presentaban estos pacientes, frente a un $38.4 \%$ que manifiestan tener un nivel de conocimientos deficiente en cuanto a las necesidades médicas que requiere este tipo de paciente. En un estudio publicado recientemente (Lewis, Freeman \& Corey, 1987), se ha podido comprobar a través de una entrevista realizada por teléfono a 1000 médicos del área de Los Angeles y de San Francisco que, fundamentalmente, la mayoría de los entrevistados no poseía el conocimiento suficiente, ni las habilidades requeridas, para asistir competentemente a pacientes con SIDA. Aquellos médicos que aparecían como competentes en este área presentaban el siguiente perfil: tenían la especialidad de medicina interna, eran médicos jóvenes, trabajaban en equipo, presentaban un nivel bajo de malestar ante el hecho de asistir profesionalmente a homosexuales, habían trabajado con pacientes con SIDA, tenían en su clínica pacientes de alto riesgo o con síntomas de SIDA, y solían advertir a determinados pacientes sobre el riesgo de infectarse con el $\mathrm{VIH}$.

Así pues, la posibilidad de tener que asistir profesionalmente a pacientes con SIDA (o seropositivos) genera una serie de estresores que fundamentalmente consisten en la posibilidad de un contagio nosocomial casual, en afrontar la situación de tener que informar acerca de la enfermedad y de sus consecuencias médicas y psicológicas, y en el impacto emocional de la muerte del paciente. Por ello, sería conveniente llevar a cabo en determinados hospitales algún tipo de programa informativo y formativo sobre modos de afrontar estos problemas. Sabemos que el peligro de contraer el SIDA nosocomialmente es muy bajo. Según Geddes (1986), ningún trabajador de la salud en todo el mundo habría contraido el SIDA de un paciente. Se han producido, eso si, algunos casos de seroconversión al VIH. En un trabajo aparecido en la revista Lancet (Anónimo, 1984), de 7270 pacientes con SIDA 
controlados por los CCE (Centros de Control de la Enfermedad) hasta el 10 de diciembre de 1984, 232 (el $3.2 \%$ ) fueron trabajadores del cuidado de la salud. No obstante, todos, a excepción de 23 (el 9.91\%), perteneclan a los denominados grupos de riesgo. La investigación epidemiológica de estas 23 personas ha podido documentar la transmisión del VIH a través de la exposición laboral. Un caso en concreto, el de una enfermera que desarrolló seroconversión entre los 28 y 49 días después de recibir una microinyección de sangre a raiz de una punción arterial, se ha documentado con precisión (Anónimo, 1984). Se han publicado, aunque no se han probado, otros 3 casos de posible transmisión del virus por pinchazos de jeringuillas, pero no se ha podido realizar su seguimiento, al negar, en dos casos, actividad de riesgo y sospecha de ella, y en el tercero no estar disponible para su control posterior (Weiss, Saxinger, Rechtman, et al. 1985). Posteriormente, se han confirmado otros tres casos de seropositividad al $\mathrm{VIH}$ a través de pinchazos accidentales con jeringuillas (Stricof \& Morse, 1986; Neisson-Vernant, Afri, Mathez, et al., 1986; Oksenhendler, Harzic, LeRoux, Rabian \& Clauvel, 1986), así como otros tres casos de infección cuya vía de transmisión se desconoce (CDC, 1987b). En los MMWR aparece además un trabajo donde de 660 sujetos (incluyendo uno que habla sido infectado de la hepatitis B) que se habian pinchado accidentalmente con jeringuillas infectadas, ninguno habla presentado seroconversión (Anónimo, 1985b). En otro trabajo (Sande, 1986), se señala que actualmente existe documentación publicada suficiente que indica que el trabajo cotidiano con pacientes infectados por el virus de inmunodeficiencia humana no implica un riesgo serio para los profesionales de la salud. De 1750 profesionales de la salud dedicados al cuidado de pacientes con SIDA, sólo en algo menos del $0.1 \%$ se encontró anticuerpos positivos del VIH (Anónimo, 1985a). En el hospital general de San Francisco se ha estudiado, durante casi 4 años, a más de 300 médicos y enfermeras que cuidaban a pacientes con SIDA; encontrándose que, a excepción de 14 de los 50 varones homosexuales trabajadores del hospital, todos los demás presentan anticuerpos negativos (Sande, 1986).

En todos los trabajos al respecto se insiste en que el riesgo de seroconversión después de un pinchazo accidental parece ser bajo. En un estudio llevado a cabo con 30 sanitarios expuestos a pinchazos de jeringuillas, $y$ en otros 55 con exposiciones de otro tipo, se encontró que ninguno presentaba anticuerpos detectables (Hirsch, Wormser, Schooley, et al. 1985). McCray, Winslow, Solomon et al. (1985), realizaron un estudio prospectivo de $\mathbf{5 0 0}$ profesionales de la salud expuestos al virus en el hospital. El $40 \%$ de estos trabajadores fueron seguidos durante un año. Ninguno desarrolló SIDA o alguna enfer- 
medad asociada al mismo. Tampoco dieron positivo a los test serológicos HTLV-III los 105 trabajadores a quienes se pasó la prueba.

Mientras que el pinchazo accidental de una jeringuilla infectada suele comportar, en el caso de la hepatitis, la transmisión del virus en el $20-30 \%$ de la veces, en el caso del SIDA parece ser que la probabilidad de infección es muy baja, menos del $0.5 \%$. Ello puede deberse a las grandes diferencias existentes en la concentración de partículas infectadas en la sangre (más de 10 elevado a 13 partículas virales por mililitro en el caso de la hepatitis, frente a 10 elevado a 4 en el caso del SIDA) (Levy, Kaminsky, Morrow, et al. 1985 ). Los resultados aportados por. Friedlan et al.(1986), así como el de Gerberdin et al. (1985), el de los CDC (1986), o los recientes de Kuhls et al. (1987) y el del CDC (1987b), corroboran las afirmaciones anteriores acerca de la dificultad de transmisión del virus a través del contacto casual, por actividades relacionadas con el trabajo asistencial a estos pacientes, incluso en personas de núcleos familiares que han permanecido en contacto estrecho con personas infectadas.

Todo lo anterior parece indicar, como conclusión, que el cuidado de pacientes con SIDA no es una actividad de alto riesgo para los profesionales de la salud. Con todo, aunque el riesgo de transmisión del $\mathrm{VIH}$ tras el pinchazo con una aguja, $u$ otra forma de exposición parenteral, es muy inferior al $1 \%$, es recomendable que se sigan cumpliendo, con rigor, las precauciones habituales para la prevención de la hepatitis $B$, o de los virus no-A no-B; así como otras relativas a la esterilización, desinfección, y eliminación de residuos; o aquellas precauciones a adoptar durante la asistencia, en su hogar,' a personas infectadas (Martin et al. 1985; CDC, 1986d; Safai, 1986; CDC, 1987a; CDC, 1987b).

Los trabajos de Lewis, Freeman \& Corey (1987), Kelly et al. (1987) y, en parte, el nuestro, sugieren que, como señala Darrow (1987), sería necesario persuadir a algunos profesionales de la salud de que el agente causal del SIDA es un virus que no se transmite por casualidad. El VIH se transmite a través de determinadas actividades específicas de riesgo: contactos sexuales sin protección, compartir jeringuillas contaminadas, a través de transfusiones de sangre contaminada, o de productos sanguíneos, $y$, perinatalmente, de una madre infectada a su hijo durante el embarazo, y quizás, durante la lactancia materna. Los trabajadores de atención en salud (T.A.S.) deben evitar pinchazos con agujas, y la exposición de otras lesiones abiertas, parenterales o mucosas, a la sangre del paciente. Para prevenir la transmisión del $\mathrm{VIH}$ desde los trabajadores de atención en salud infectados y con lesiones exudativas a los pacientes, la medida más importante consiste en evitar un contacto directo con el mismo. Evi- 
tar la transmisión paciente a paciente exige una rigurosa esterilización del equipo reutilizable, tal como agujas, jeringas, e instrumentos penetrantes en la piel (OMS, 1987).

Una gran parte de la solución del SIDA reside en una rápida difusión de información importante y precisa. Aunque todavía desconocemos muchas variables (grado de susceptibilidad personal a la infección, incidencia y prevalencia real, terapéutica eficaz, vacuna, entre otras muchas), las tasas de incidencia siguen aumentando en todo el mundo, aunque con una intensidad mucho menor que la estimada inicialmente, por lo que se requiere un gran esfuerzo de cooperación multi-interdisciplinar. En este sentido, los psicólogos de la salud podemos desempeñar un papel importante no sólo en el diseño y ejecución de programas educativos acerca de las actividades de riesgo de infección de VIH sino también en la realización de programas específicos para trabajadores de atención en salud.

Nota 1: Hasta el 26 de Junio de 1987, según el Servicio de Información Epidemiológica del Ministerio de Sanidad y Consumo el número de casos de SIDA en la Provincia de Alicante era de 4.

\section{REFERENCIAS}

Ancelle-Park, R., Brunet, J. \& Downs, A.(1987): AIDS and Drug Addicts in Europe. The Lancet, 626-627.

Anónimo (1984): Needlestick transmission of HTLV3 from a patient infected in Africa (Editorial). Lancet, 2: 1376-1377.

Batchelor, W. (1984): AIDS: a public health and psychological emergency. American Psychologist, 39 (11), 1279-1284.

Bayés, R. (1986): ¿Podemos aportar algo los psicólogos conductuales a la solución del problema del SIDA? Papeles del Cologio de Psicólogos, 27, 49-53.

Blumenfield, M.,Smith, P.,Milazzo, J., Seropian, S. \& Wormser,G. (1987): Survey of Attitudes of Nurses Working with Aids Patients. General Hospital Psychiatry, 9, 56-63.

Centers for Disease Control (1985a): Update: evaluation of human T-lymphotropic virus type III/ lymphadenopathy-associated virus infection in health -care personnelUnited States. MMWR, 34, 575- 578. 
Centers for Disease Control (1985b): Summary: recommendations for preventing transmission of infection with human T-limphotropic virus type III/lymphadenopathy-associated virus in the workplace. MMWR, 34, 681-685, 691-695.

Centers for Disease Control (CDC) (1985c): Recommendations for assisting in the prevention of HIV and AIDS. MMWR ,Dec. 6.

Centers for Disease Control (CDC)(1985d): Recommendations for preventing transmission of HTLV-III/LAV by ocular fluids. MMWR, August, 30.

Centers for Disease Control (CDC) (1986a): Additional recommendations to reduce sexual and drug abuse-related transmission of HTLV-III/LAV.MMWR, Feb. 14.

Centers for Disease Control (CDC) (1986b): Recommendations for preventing transmission of infection with HTLV-III/LAV during invasive procedures.MMWR, 35, 221-223.

Centers for Disease Control (CDC)(1986c): Recommended infection-control practices for dentistry. MMWR, 35, 237-242.

Centers for Disease Control (CDC) (1986d): Human T-Lymphotropic Virus Type III/ Lymphadenopathy-Associated Virus: Agent Summary Statement. MMWR, vol.35 (34), 540-549.

Centers for Disease Control (CDC) (1987a): Human immunodeficiency virus Infection in Transfusion Recipients and their Family Members, MMWR,36 (10), 137140.

Centers for Disease Control (CDC) (1987b): Update: Human Immunodeficiency Virus Infections in Health-Care Workers Exposed to Blood of Infected Patients. MMWR, 36, 285-289.

Coates, Th., Temoshok, L. \& Mandel, J. (1984): Psychosocial Research is essential to understanding and treating AIDS. American Psychologist, 39(11), 1309-1314.

Darrow, W. (1987): A Framework for Preventing AIDS. American Joumal of Public Health, 77(7), 778-779.

Delgado, A. (1988): Manual S.J.D.A. Aspectos Médicos y Sociales. Organización Médica Colegial.

Detmer, W. \& Lu, F. (1986-87): Neuropsychiatric complications of AIDS: a literature review. Intemational Joumal of Psychiatry in Modicine, 16, 21-32.

Douglas, C., Kalmann, C. \& Kalman, Th. (1985): Homophobia Among physicians and nurses:an empirical study. Hospital \& Community Psychiatry ,36(12), 1309-1311.

Duvic, M., Rios, A. \& Brewton, G. (1987): Aids and drug addicts in Europe.The Lancet,sept. 12, 626-627.

Ferrara, A. (1984): My personal experience with AIDS. American Psychologist , 39 (11), 1285-1287.

Friedland, GH., Saltzman, BR., Rogers, MF. et al. (1986): Lack of transmission of HTLV-III/LAV infection to household contacts of patients with AIDS or AIDS-related complex with oral candidiasis. New England Journal of Medicine, 314, 344-349. 
Fonseca, E. (1988): Sidofobia: una nueva faceta de un viejo problema.Med. Clin (Barc), 90, 131-132.

Gallo, R. (1987): 'El primer retrovirus humano. Investigación y Ciencia, 125, 44-55.

Gallo, R. (1987): El virus del SIDA. Investigación y Ciencia, 126, 31-41.

Gascón, P. (1987): Impacto del SIDA en el personal médico. Jano, XXXIII(782), 393-394.

Gebberding, JL, Hopewell, PC., Kaminsky, LS., \& Sande, MA. (1985): Transmission of hepatitis B without transmission of AIDS by accidental needlestick. New England Joumal of Medicine, 312, 56.

Geddes, A.M. (1986): Risk of AIDS to health care workers. British Medical Joumal, 292 (6522),711-712.

Goedert, J.J. \& Blattner, W.A. (1986): Epidemiología del SIDA y trastornos asociados. En V. T. DeVita...(op. cit.).

Gold, M., Seymour, N. \& Sahl, J. (1986): Counseling HIV Seropositives. En L. McKusick (ed.): What to do about AIDS. Physicians and Mental Health Professionals Discuss the Issues, Univ. of California Press.

Hardy, A., Ranch, K., Echenberg, D., Morgan, W. \& Curran, J. (1986): The economic impact of the first 10.000 cases of Acquired Immunodeficiency Syndrome in the United States. JAMA, 255 (2), 209-211.

Horstman, W. \& McKusick, L. (1986): The impact of AIDS on the Physician. En L. Mckusick (ed.): What to do about AIDS. Physicians and mental Health Professionals Discuss the /ssues. Univ. of California Press.

Kelly, J. St.Lawrence, J., Smith, S., Hood, H. \& Cook, D. (1987): Stigmatization of AIDS Patients by Physicians. American Joumal of Public Health, 77(7), 789-791.

Kelly, J., St.Lawrence, J., Smith, S., Hood, H. \& Cook, D. (1987): Student' Attitudes toward AIDS and Homosexual Patients. J. of Medical Education, 62(7), 549-556.

Kuhls, Th., Viker, S., Parris, N., Garakian, A., Sullivan-Bolyai, J., \& Cherry, J. (1987): Occupational risk of HIV, HBV and HSV-2 Infections in Health Care Personnel Caring for AIDS Patients. American Joumal of Public Health. 77(10), 1306-1309.

Leal, M. \& Flores, J.M. (1987): Infección por el virus de la inmunodeficiencia humana en la infancia: la cara oculta de la drogadicción parenteral. Med. Clin. (Barc.), 89, $689-691$.

Lewis, C., Freeman, H., Kaplan, S. \& Corey, C.(1986): The impact of a program to enhance the AIDS-related competencies of primary care physicians. Journal of General Internal Medicine, 1: 287-294.Lewis, C., Freeman, H. \& Corey, C. (1987): AIDS-related competence of California's Primary care Physicians.American Journal of Public Health, 77(7), 795-799.

Martin,J. \& Vance, C. (1984): Behavioral and Psychosocial factors in AIDS. Methodological and Substantive Issues. American Psychologist, 39(11), 1303-1308.

Mathews, W. Both, M. Turner, J. \& Kessler, L. (1986): Physicians' attitudes to- 
wards homosexuality: Survey of California Medical Society.West Joumal of Medicine, 144, 106-110.

Morin, S. Charles, K., \& Malyon, A. (1984): The psychological impact of AIDS on Gay Men. American Psychologist, 39 (11), 1288-1293.

Neisson-Vernant, C., Afri, S., Mathez, D., et al. (1986): NeedlesticK HIV seroconversion in a nurse. Lancet,2, 814.

O'Donnell, L., O'Donnell, C.R., Pleck, J.H., Snarey, J.K. \& Rose, R.M. (1987): Psychosocial Responses of Hospital workers to Acquired Immune Deficiency Syndrome (AIDS). Joumal of Applied Social Psychology, 17(3), $269-285$.

Oksenhendler, E., Harzic, M., LeRoux, J., Rabian,C., \& Clauvel, J. (1986): HIV infection with seroconversion after a superficial needlestick injury to the finger. New England Joumal of Medicine, 315, 582.

Organización Mundial de la Salud (1987): Recomendaciones para la prevención y control de infección con VIH. Ministerio de Sanidad y Consumo. Dirección General de Planificación Sanitaria (orig. en inglés, 1986).

Reig, A. y Caruana, A. (1987): Bateria de Evaluación de Estrés Ocupacional en Profesionales de la Salud. Mimeo. Depto. Psicología de la Salud.

Safai, B. (1986): Precauciones que deben adoptarse al atender casos de SIDA. En V.T. DeVita, S. Hellman \& S.A. Rosenberg (eds): SIDA. Etiologia, diagnóstico, tratamiento y prevención. Salvat.

Sande, M.A. (1986): Transmission of AIDS. The case against casual contagion. The New England Joumal of Medicine, 314 (6), 380-282.

Selwyn, P. (1987): Estado actual de los conocimientos sobre el SIDA (IV). Aspectos psicosociales, perspectivas terapéuticas. Hospital Practice (Ed. español), 2(7), 69-86.

Stricof, RL. \& Morse, DL. (1986): HTLV-Ill/LAV seroconversion following a deep intramuscular needlestick injury. New England Journal of Medicine, 314, 1115.

Tor, J. (1987): III Conferencia Internacional sobre el Síndrome de Inmunodeficiencia Adquirida (SIDA). Jano, XXXIII(783), 481-484.

Vaqué, J. \& Arcalís, L. (1987): La problemática del personal sanitario seropositivo al virus de la inmunodeficiencia humana: entre la confidencialidad y el riesgo. Med. Clin. (Barc), 89, 331-334.

Wachter, R. (1986): The impact of the acquired inmunodeficiency syndrome on medical residency training. The New England Joumal of Medicine, 314(3), 177-180.

Weiss, SH., Saxinger, WC., Rechtman, D. et al. (1985): HTLV-III infection among health care workers: association with needlestick injuries. JAMA, 254, 2089-2093. 